\title{
INÉDITOS Y OTRAS CONSIDERACIONES SOBRE PAOLO DE MATTEIS, GIUSEPPE SIMONELLI, LORENZO RUGGI Y OTROS SEGUIDORES DE LUCA GIORDANO
}

Data recepción: 2010/09/10

Data aceptación: 2011/07/13

Contacto autor: mdimauro@unina.it
Marco di Mauro

Universitá di Napoli

\begin{abstract}
RESUMEN
Cientos de pinturas napolitanas de los siglos XVII y XVIII han sido generalmente atribuidas a la escuela de Luca Giordano, quien tuvo un largo número de alumnos cuyos nombres han sido transmitidos por las principales fuentes de la historiografía artística. A algunos de ellos, como Franceschitto o Monsù Anselmo, no les podemos asociar ni siquiera con una obra. Por el contrario, hay casos de seguidores anónimos de Giordano con un estilo reconocible, como el autor de las dos versiones de Santiago el Mayor, una conservada en Casalnuovo de Nápoles y la otra en Lecce. Otras obras siguen fielmente el estilo de Giordano y llevan la firma de pintores que serían de otro modo desconocidos, caso de Lorenzo Ruggi, que firma una hermosa Inmaculada en la iglesia de S. Francisco de Aversa. Otro notable artista seguidor de Giordano es el «G. Fatteruso» que firma el Milagro de San Biagio en la iglesia de San Biagio en Mugnano de Nápoles. Es sin duda plausible la identificación con Giuseppe Fattorusso, recordado como discípulo de Vaccaro y luego de Beinaschi, si bien la comparación con sus obras documentadas plantea algunas dudas. Por último, una revisión de obras de diversa calidad, atribuidas con reserva a Luca Giordano o a su taller, puede arrojar una nueva luz sobre la cuestión de las numerosas imitaciones de las obras del maestro. La calificación de copias sería la más adecuada, por ejemplo, para las dos versiones de la Bendición de Isaac aparecidas en el mercado anticuario de Nueva York y en una colección privada en Sant'Arpino.
\end{abstract}

Palabras clave: Nápoles, Luca Giordano, Paolo de Matteis, Giuseppe Simonelli, Giuseppe Fattorusso

ABSTRACT

Hundreds of 17th- and 18th-century Neapolitan paintings have been generically attributed to the school of Luca Giordano, who had many pupils, whose names have become known to us thanks to the leading historiographical sources of Neapolitan art. The work of some of these artists, such as Franceschitto and Monsu Anselmo, is unknown, although there are some anonymous Giordano followers who can easily be identified by their style, among them the author of two versions of St James the Greater, one of which is kept in Casalnuovo di Napoli and the other in Lecce. In addition, there are other works that are heavily influenced by Giordano and signed by hitherto unknown artists. These include Lorenzo Ruggi, who painted the wonderful Immaculate adorning St Francis Church in Aversa. Recent documentary research has revealed some information on the painter. Another leading follower of Giordano's is one "G. Fatteruso", who completed the majestic Miracle of St Blaise at St Blaise Church in Mugnano di Napoli. Though there are obvious grounds for identifying this artist as Giuseppe Fattorusso, remembered as a pupil of Vaccaro and later of Beinaschi, comparisons with the latter's documented works suggest that this is not the case. Finally, newly conducted analysis of poorer-quality paintings that are cautiously attributed to Giordano or his studio, has enabled more detailed investigation into the problem regarding copies of Giordano's famous works. Examples of this are two depictions of The Blessing of Isaac, one sold in New York and another in Sant'Arpino (near Naples), both of which can only be regarded as copies of a lost Giordano composition.

Keywords: Naples, Luca Giordano, Paolo de Matteis, Giuseppe Simonelli, Giuseppe Fattorusso 
Los discípulos, seguidores e imitadores de Luca Giordano de los que se tiene constancia son innumerables. De Dominici' menciona decenas de alumnos. Algunos, como Franceschitto o Monsù Anselmo, todavía no han sido identificados al no conservarse sus obras. Otros pintores seguidores de Giordano son conocidos únicamente por las firmas encontradas en los lienzos y en los frescos, después de restauraciones o limpiezas que han removido el polvo que las ocultaba. Recordamos entre estos pintores a Lorenzo Ruggi, que firma y fecha en 1671 una tela con la Inmaculada en la iglesia de S. Francesco ad Aversa (Fig. 1). Sin embargo, la fecha de esta obra no deja de causar cierta perplejidad, ya que la pintura deriva de modelos que imitan a Giordano, como la Inmaculada del Museo de Arte de Ponce o la del Palacio Pitti de Florencia (Fig. 2), ambas datadas alrededor de 1680, e introduciendo sutiles aperturas clasicistas².

La obra de Lorenzo Ruggi manifiesta su descendencia de los modelos de Giordano en la tierna carnación de los amorcillos, en las brillantes vestiduras de María, en la luz dorada que irrumpe entre las nubes, en la vitalidad de la composición acentuada por la alegre agrupación de los amorcillos. Es por tanto verosímil que Lorenzo Ruggi fuera un "alumno de Giordano", como afirma Parente ${ }^{3}$, aunque De Dominici no le nombra entre sus discípulos. Labrot ofrece más noticias $^{4}$, citando, sobre la base de los documentos de archivo, obras de Lorenzo Ruggi presentes en los inventarios de dos colecciones napolitanas: la del doctor Antonio Lauro, su hermanastro, y la del príncipe de Montefalcone, Andrea Coppola. Ruggi, fallecido en 1687, se dedicó mayoritariamente a pintar temáticas sagradas, pero también paisajes de los que, sin embargo, no ha quedado huella. Labrot supone que habría muerto en plena actividad, ya que al momento de su fallecimiento se encontraban muchos cuadros aún sin vender en su tienda.

A diferencia de Ruggi, la figura de Giuseppe Simonelli sustenta una conspicua bibliografía y centenares de obras firmadas o atribuidas. Entre los últimos hallazgos se encuentra la Apoteosis de S. Juan Evangelista de la iglesia de S. Giovanni en Teverola (Fig. 3). Hasta el año 1989, cuando la tela fue retirada del techo de la nave para su restauración, la obra se había atribuido a un desconocido napolitano, pero el descubrimiento de la firma, abajo a la derecha, no deja duda alguna sobre la paternidad de Giuseppe Simonelli. Aquí el pintor de Aversa utiliza muchos modelos de Giordano, como la Apoteosis de Júpiter con Diana y Apolo de la colección Denis Mahon de Londres ${ }^{5}$ (Fig. 4), recordando el quehacer pictórico ligero y vibrante del maestro.

Además, se tiende a atribuir a Simonelli cualquier pintura que siga fielmente a Giordano, cuando presenta una alta calidad y, generalmente, una preeminencia de los tonos oscuros. Una obra que manifiesta estos caracteres es el Sermón de Santiago el Mayor (datada alrededor de 1680-1685), custodiada en la iglesia de S. Giacomo en Lecce (Fig. 5). El lienzo fue dado a conocer por Mimma Pasculli ${ }^{6}$, que la atribuyó de modo hipotético a Simonelli en base a la confrontación primaria con la tela de análogo tema (1697) de la iglesia napolitana de S. Caterina de Formiello (Fig. 6). Sucesivamente Mariaclaudia Izzo propuso un cambio de atribución a favor de Nicola Vaccaro ${ }^{7}$, para luego cambiar de opinión y confirmar la referencia de Pasculli a Giuseppe Simonelli. Una ulterior revisión, la que aquí se propone, me inclina a rechazar tanto una como otra atribución, en favor de un desconocido seguidor de Giordano. La confrontación inédita que se aporta ahora es con el sermón de Santiago situado sobre el altar mayor de la iglesia de S. Giacomo en Casalnuovo de Nápoles (Fig. 7), que es una réplica con variantes de la tela de Lecce: la figura del santo es muy parecida, mientras que, en los rostros y en las poses, un poco rígidas de los asistentes, se advierten algunas debilidades no imputables a un pintor refinado como Simonelli. Se pueden notar análogas debilidades en la tela de Lecce, que probablemente debería atribuirse al mismo autor. Contrariamente, la tela de Giuseppe Simonelli en $\mathrm{S}$. Caterina de Formiello manifiesta una alta calidad tanto en la figura del santo como en las de los asistentes, bien caracterizados en la expresión de los rostros y en la variada gestualidad. Mi conclusión es que el sermón de Santiago en Lecce y la de Casalnuovo son obras de un desconocido seguidor de Giordano que, como Simonelli en el lienzo napolitano, replicó la figura central de un modelo perdido de Luca Giordano. 
Se conservan centenares de copias de obras de Giordano, algunas de calidad decididamente modesta, y que por tanto se pueden considerar como ejercicios de taller, y otras de calidad más alta, que pueden hacer suponer una intervención directa del maestro. Recientemente han visto la luz dos réplicas de una perdida Bendición de Jacob de Luca Giordano: una, que ha aparecido en el mercado anticuario de Nueva York, ha sido analizada en una publicación de Stefano Causa ${ }^{9}$ y atribuida a Luca Giordano y a un colaborador; la otra ${ }^{10}$, que he localizado en una colección particular en Sant'Arpino (Fig. 8), es ciertamente una obra de taller.

Las dos telas representan a Jacob que consigue la bendición de Isaac, en presencia de la madre Rebeca que ha tramado el engaño. A la izquierda, algunas mujeres se apresuran a cocinar el cabrito que Isaac ha pedido al primogénito Esaú, el cual, en las intenciones paternas, estaba destinado a recibir la bendición (Génesis, 27). La elección iconográfica, del todo inusita$\mathrm{da}$, que representa la escena de la cocina junto con la escena de la bendición, nace probablemente de un encargo de tipo laico y particular.

La composición de la obra ha sido muy estudiada, considerando el recurso de la columna sobre plinto que divide el primer plano, la escena de la bendición, en segundo plano, o la escena de la cocina. Un recurso, este último, que recorre frecuentemente en la pintura napolitana, de Giordano a Solimena, pero que encuentra sus raíces en la pintura veneciana y particularmente en la célebre Pala Pesaro de Tiziano.

Respecto a la tela de Nueva York, pienso que es necesario invertir la atribución: no tanto obra de Giordano y colaborador, como obra de taller con la vaga posibilidad de una intervención de Giordano. De otra manera no sería justificable la modesta calidad del rostro de Jacob, alrededor del cual gira toda la composición.

Las debilidades presentes en la tela de Nueva York son todavía más evidentes en la tela de Sant'Arpino, donde las figuras de los protagonistas, Isaac y Jacob, carecen de vigor y de expresividad. Están más conseguidos el rostro de Rebeca, con el áspero encarnado envuelto en la sombra, y la escena de la cocina, con las figuras delineadas a través de una redacción sintética y abreviada, aunque densa, conseguida con rápi- dos apoyos de color denso y luminoso. Un cuidado particular se dedicó también a las figuras de los perros, muy frecuentes en las composiciones de Luca Giordano, que confieren una alegre entonación cotidiana.

El engaño tramado por Rebeca a costa de Esaú recuerda al engaño existente con las réplicas de Luca Giordano, que frecuentemente, también de buena fe, permiten atribuir al maestro obras de calidad no tan elevada. Ya en los tiempos de De Dominici circulaban muchas réplicas de Giordano, como las de Giuseppe Simonelli, que no tan raramente "engañaban a los forasteros" $" 11$.

Otra obra de taller que, en el archivo fotográfico de la Soprintendenza alle Gallerie de Nápoles (neg. 38877), se encontraba atribuida impropiamente a Luca Giordano, era la Adoración de los pastores del Museo Diocesano de Nápoles $^{12}$ (Fig. 9). La tela se puede adscribir, más bien, a un pintor napolitano que sigue la producción más cercana a Bassano del propio Giordano, como la Adoración de los pastores de la colección Rossi de Forlì ${ }^{13}$, que tiene una datación alrededor de 1682-1686, y la siguiente versión custodiada en la Annunziata de Gaeta ${ }^{14}$. Es más propiamente en esta fase cuando Luca Giordano se aleja del barroco de Cortona y del gigantismo de Tiziano y Veronés, para dirigir sus intereses hacia otro filón de la pintura veneciana, aquella más dulce e íntima de Jacopo da Bassano.

Si la versión pictórica del tema recuerda, por lo tanto, a los modos de Bassano asimilados por el propio Giordano, en cambio su composición calca la Adoración de los pastores de Guido Reni del coro de la cartuja de S. Martín. Un modelo, éste, imprescindible para los pintores napolitanos del siglo XVII, fascinados por la profunda y sincera representación de los afectos que Reni había aprendido en la escuela de los Carracci.

Actualmente no es posible individualizar, entre los numerosos seguidores de Giordano, al autor de la tela del Museo Diocesano. Sin embargo, la preeminencia de los tonos marrones y las aperturas que siguen a Bassano pueden recordar al Giuseppe Simonelli (1649-1713) del periodo de la Sagrada Familia, obra perteneciente a una colección particular (subasta Sotheby's de Londres, 9 de diciembre de 2004, lote 189). 


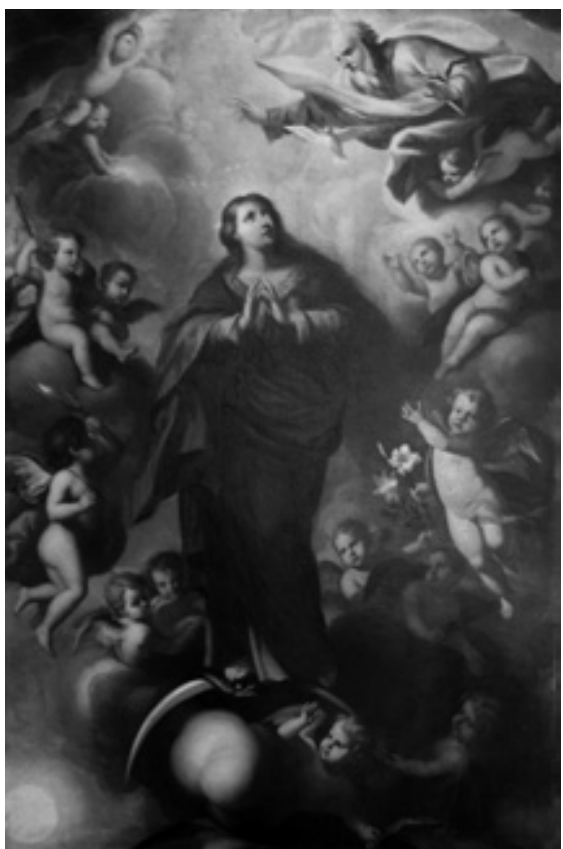

Fig. 1. Lorenzo Ruggi, Inmaculada. Aversa, iglesia de S. Francesco.

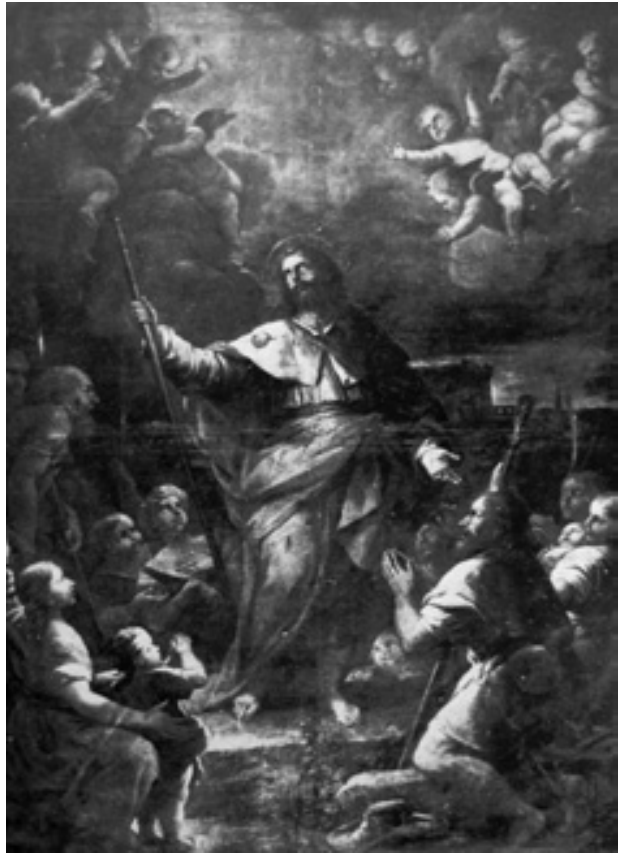

Fig. 5. Seguidor desconocido de Giordano, Sermón de Santiago el Mayor. Lecce, iglesia de S. Giacomo.

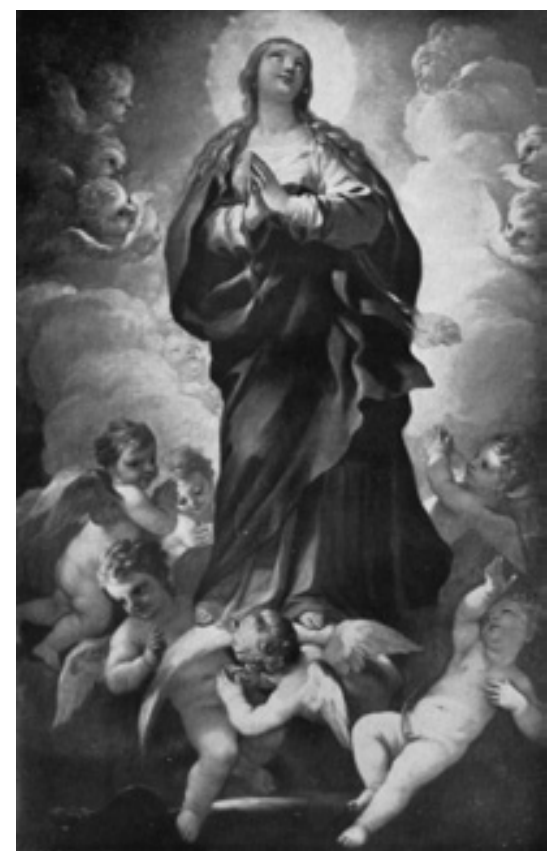

Fig. 2. Luca Giordano, Inmaculada. Florencia, Galleria di Palazzo Pitti.

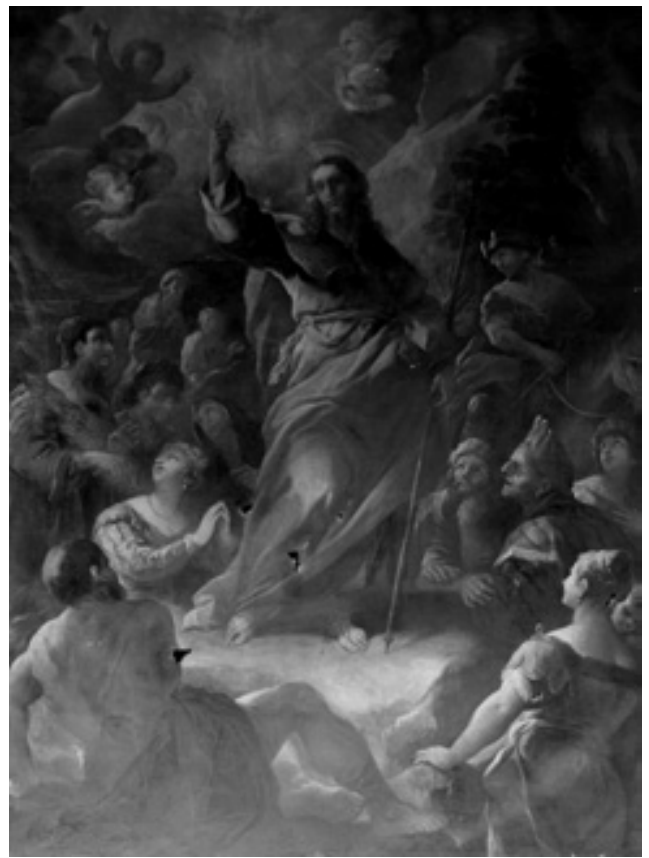

Fig. 6. Giuseppe Simonelli, Sermón de Santiago el Mayor. Nápoles, iglesia de S. Caterina a Formiello. 


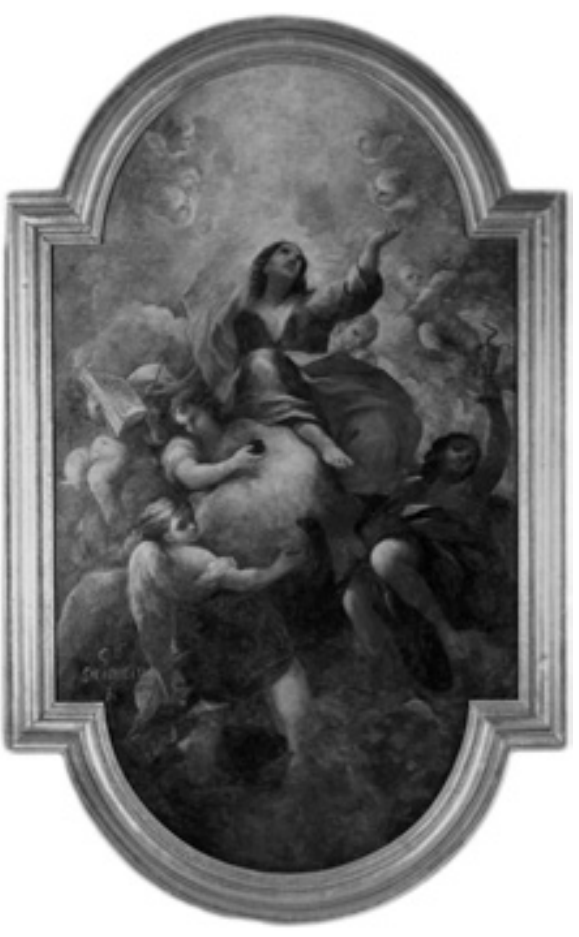

Fig. 3. Giuseppe Simonelli, Apoteosis de San Juan Evangelista. Teverola, iglesia de S. Giovanni.

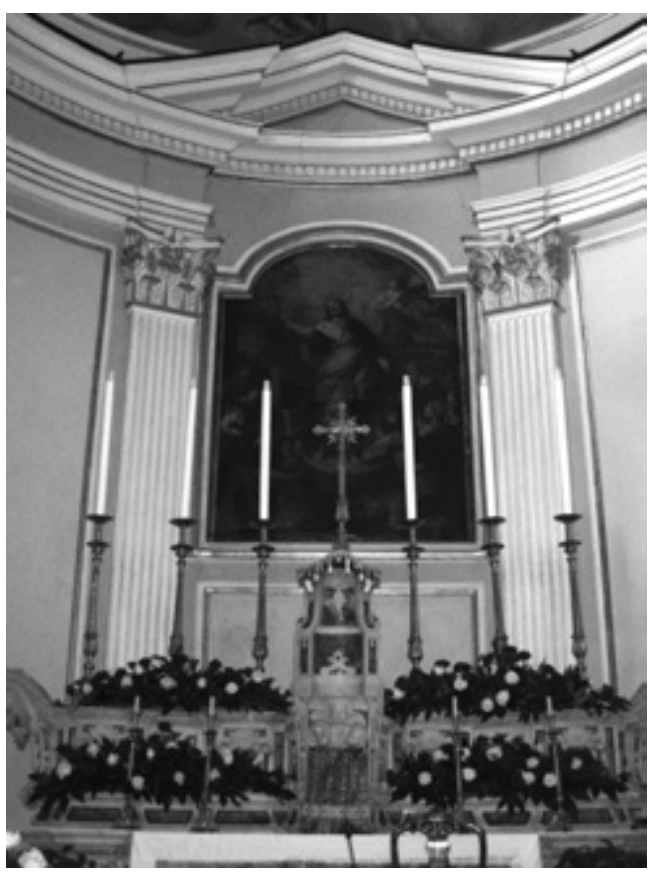

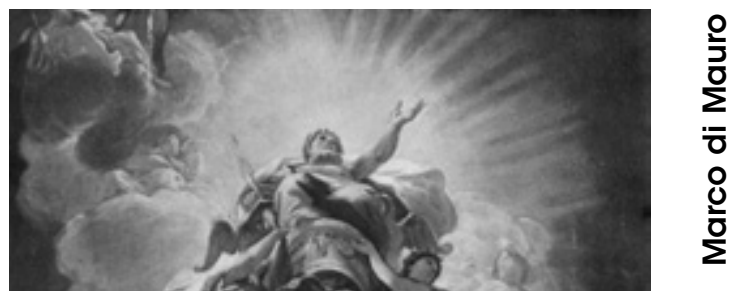

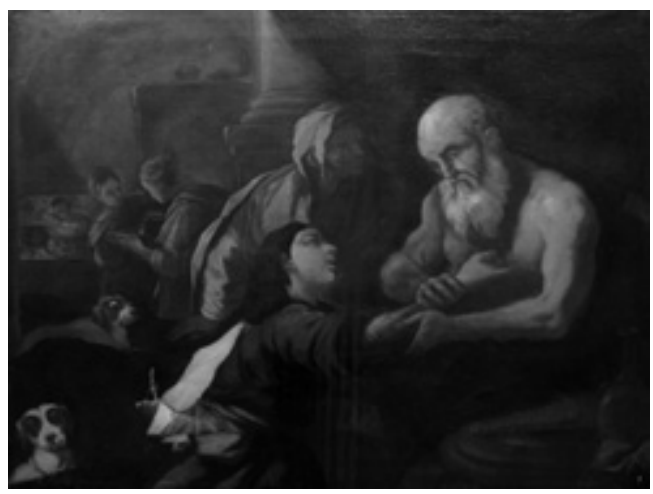

Fig. 8. Seguidor desconocido de Giordano, Bendición de Jacob. Sant'Arpino, colección privada.

4 Fig. 7. Seguidor desconocido de Giordano, Sermón de Santiago el Mayor. Casalnuovo de Nápoles, iglesia de S. Giacomo. 


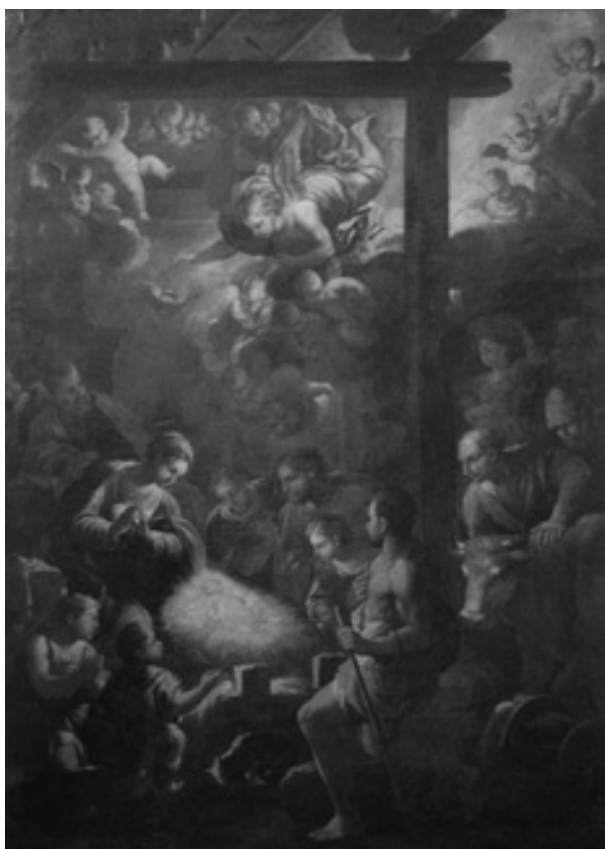

Fig. 9. Seguidor desconocido de Giordano, Adoración de los pastores. Nápoles, Museo Diocesano.

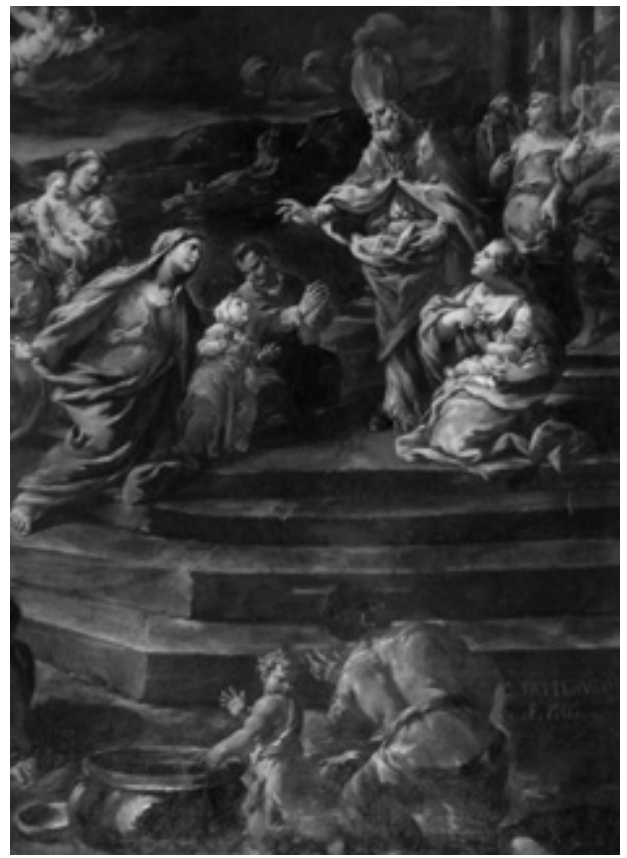

Fig. 13. G. (Giuseppe?) Fattorusso, Milagro de S. Biagio. Mugnano de Nápoles, iglesia de S. Biagio.

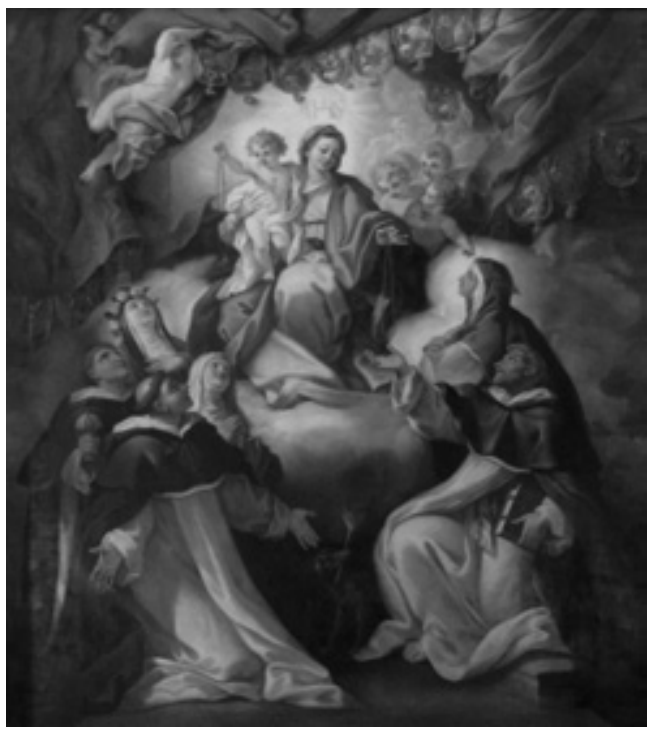

Fig. 10. Atribuido a Paolo de Matteis, Madonna del Rosario. Mugnano de Nápoles, iglesia de S. Biagio.

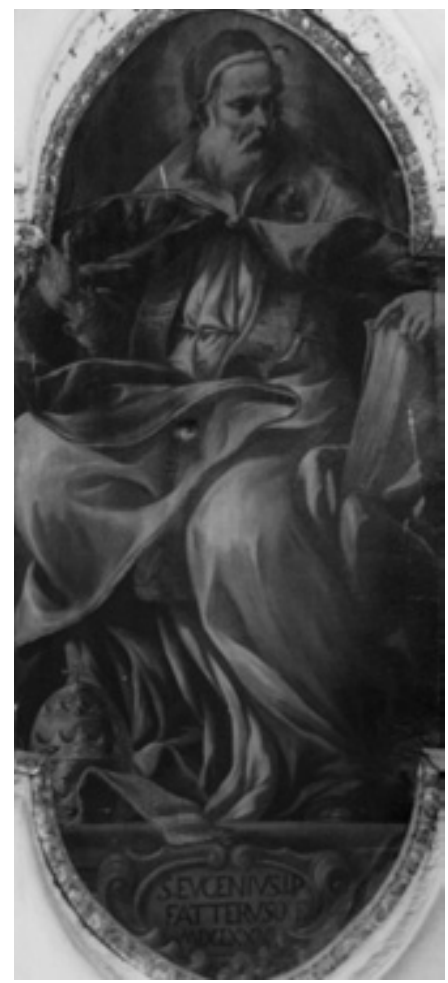

Fig. 14. Giuseppe Fattorusso, S. Eugenio I papa. Nápoles, iglesia de Regina Coeli. 


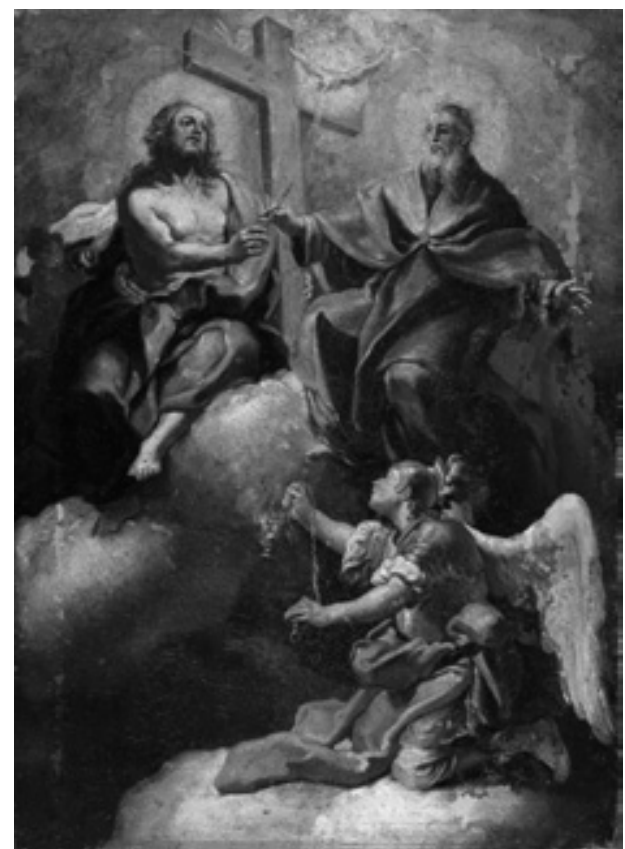

Fig. 11. Domenico Antonio Vaccaro, Trinidad con San Miguel Arcángel. Ariccia, Museo del Barocco Romano (foto: Daniele Petrucci).

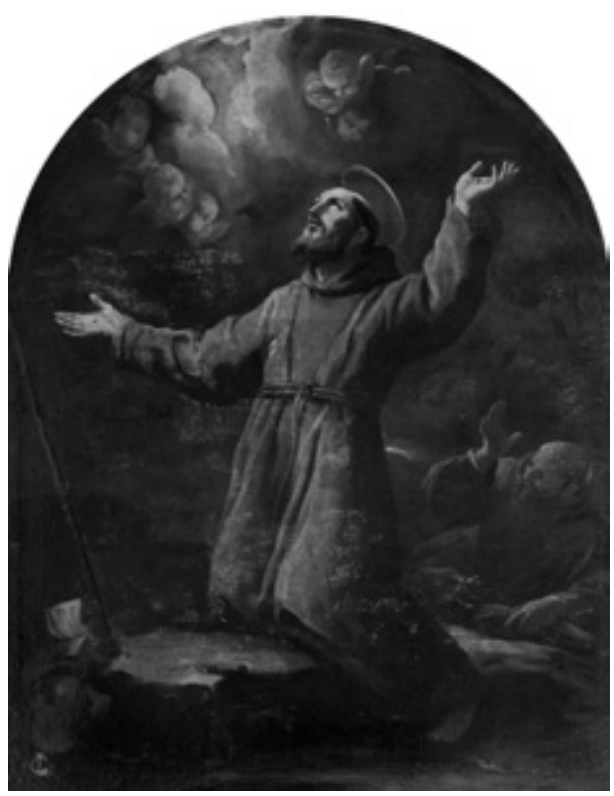

Fig. 15. Seguidor desconocido de Giordano, Éxtasis de S. Francisco de Asís. San Sebastiano al Vesuvio (Nápoles), capilla de Villa Figliola.

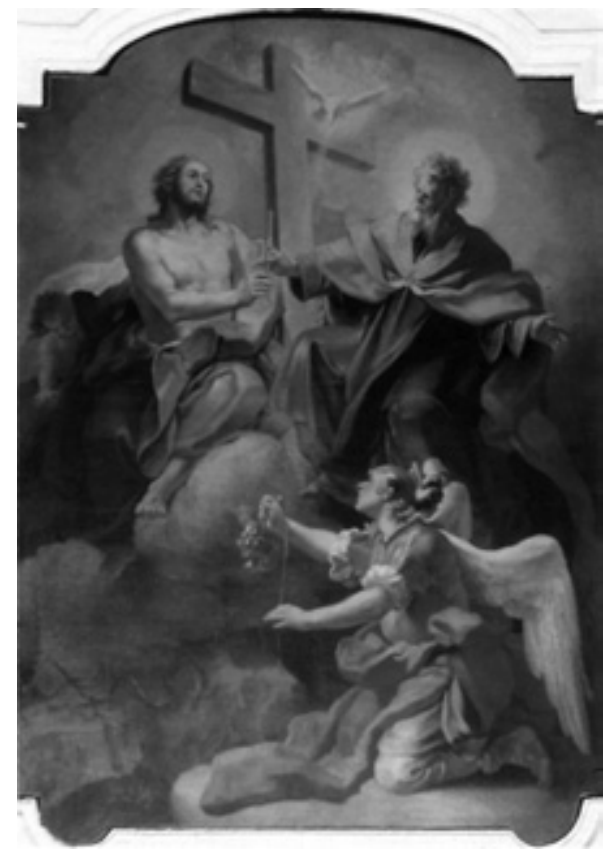

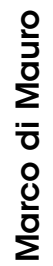

Fig. 12. Domenico Antonio Vaccaro, Trinidad con San Miguel Arcángel. Nápoles, iglesia de la Concezione a Montecalvario.

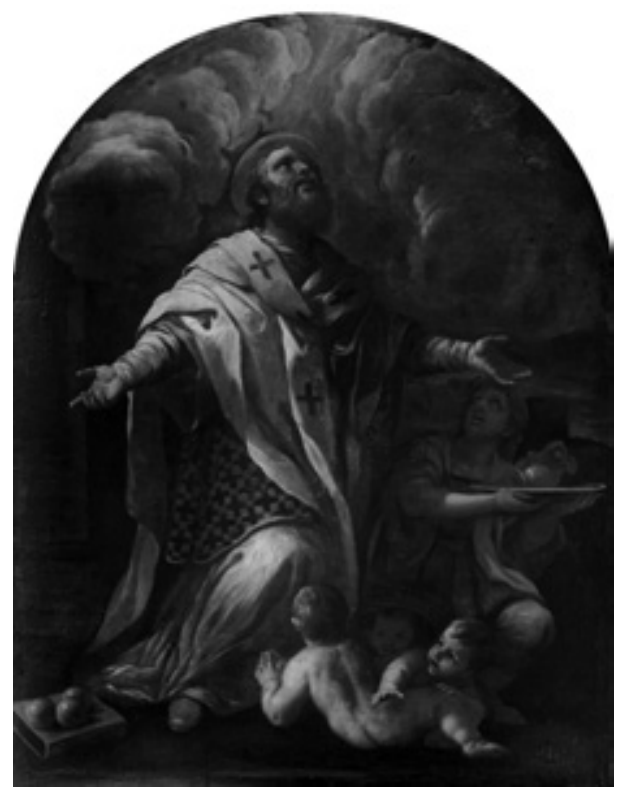

Fig. 16. Seguidor desconocido de Giordano, S. Nicolás de Bari con el copero Basilio. San Sebastiano al Vesuvio (Nápoles), capilla de Villa Figliola. 
Un discípulo de Giordano que, a diferencia de Simonelli, supo distinguirse del maestro elaborando un lenguaje propio y reconocible, con una clara huella clasicista, es el pintor originario del Cilento Paolo de Matteis (1662-1728). Una notable Virgen del Rosario, custodiada en la iglesia de S. Biagio de Mugnano de Nápoles, se puede atribuir a de Matteis (Fig. 10). De hecho ya fue estudiada en una publicación anterior, con una cauta atribución a un "valioso discípulo de Paolo De Matteis ${ }^{15}$. Después de ulteriores investigaciones sobre el cuadro, considero que puedo arriesgar una atribución al propio de Matteis, por la complejidad de la composición y la elevada calidad pictórica, que sufre solo una leve degradación en las fisonomías de los amorcillos, donde es probable la intervención del taller. A los pies de la Virgen se encuentran seis figuras de santos dominicos: a la izquierda S. Tomás de Aquino, S. Jacinto, S. Rosa de Lima y $\mathrm{S}$. Catalina de Ricci (?); a la derecha S. Domingo de Gúzman y S. Catalina de Siena.

La fastuosa y monumental composición, con los Misterios del Rosario pintados a lo largo de los bordes de la cortina, está inspirada en la Virgen del Baldaquín de Luca Giordano, hoy en Capodimonte, de la cual, sin embargo, se aleja por el encuadre más próximo, la claridad de los tintes y la tendencia moderadamente clasicista, con una sutil entonación sentimental. Son los elementos que de Matteis aprendió en Roma, después de conocer a Carlo Maratta, y que desarrolló en París en los años 1702-1705, cerca de la corte de Luís XIV. Aí trabajaba Charles de La Fosse, que había sido alumno de Charles Le Brun, al cual Mario Alberto Pavone ${ }^{16}$ atribuye la renovación estilística de De Matteis. En efecto, a su regreso a Nápoles el pintor propuso un clasicismo dulce y elegante, de tiernos colores pasteles, sugiriendo una alternativa al clasicismo más destacado y solemne propugnado entonces por Francesco Solimena.

Por todos los elementos que hemos subrayado, la Virgen del Rosario de Mugnano tiene que ser encuadrada en la última producción de De Matteis, alrededor del año 1720, en sintonía con el ciclo de frescos de Guardia Sanframondi, donde también se nota la intervención del taller en las figuras marginales.
Una ulterior comparación se puede establecer con la Virgen del Rosario de Antonio y Giovanni Sarnelli, identificados entre los más fieles seguidores de De Matteis, presente en la iglesia de la Magdalena de Morano Calabro. Los Sarnelli pintan alrededor de la Virgen una especie de baldaquino con los Misterios del Rosario, con una solución de la composición sustancialmente análoga ${ }^{17}$.

Otra obra a considerar es una pintura de Domenico Antonio Vaccaro, que se formó en el taller de Solimena y que se opuso brillantemente a las intenciones puristas del maestro, elaborando sugestiones que recuerdan a Giordano pero en clave rococó. La obra que se trae a colación es una apreciable Trinidad con S. Miguel Arcángel (Fig. 11), entonces en la colección Ferrari, hoy en el Museo del Barroco Romano de Ariccia. La atribución de la tela a Domenico Antonio Vaccaro es mérito de Vincenzo Pacelli ${ }^{18}$, con una datación alrededor del cuarto decenio del siglo XVIII.

Hoy, a la luz de recientes investigaciones sobre esta obra, puedo afirmar que se trata de un boceto para la tela de análogo tema conservada en Nápoles, en la iglesia de la Conzecione de Montecalvario (Fig. 12). La única diferencia entre el boceto y la obra acabada reside en la presencia del grupo de almas en la parte inferior izquierda, que nos recuerda la tarea, asignada a S. Miguel Arcángel, de cribar las almas antes del juicio. El reconocimiento de esta obra implica anticipar la datación alrededor del año 1720, justificando así la vivacidad de la composición y la brillantez pictórica que se encuentra frecuentemente en la producción juvenil de Vaccaro, por ejemplo en la Alegoría del papado de Clemente $X I$ de la Walter Art Gallery de Baltimore ${ }^{19}$.

Finalmente, podemos retornar a la iglesia de S. Biagio de Mugnano de Nápoles para observar el espléndido Milagro de S. Biagio (Fig. 13) que domina el altar mayor. La tela, firmada «G. Fatteruso F. 1707», ofrece una notable aportación para la comprensión de Giuseppe Fattorusso, pintor escasamente conocido si no fuera por haber colaborado en algunas obras de Giovan Battista Beinaschi. Efectivamente, la dependencia estilística de Beinaschi es el único dato que emerge de su producción conocida, pero en la pintu- 
ra de Mugnano, Fattorusso revela claramente la influencia de Luca Giordano en la pastosidad de las pinceladas y en algunas soluciones formales, como la figura de espaldas en primer plano, que recuerda a uno de los soldados del Martirio de $S$. Gennaro en Roma (iglesia de S. Spirito dei Napolitani). También la concepción de la composición, con la visión en escorzo de la gradería que sube al templo, puede confrontarse con modelos de Giordano como la Presentación de la Virgen al Templo del Kunsthistorisches Museum. Es probable que el repentino cambio de rumbo de la pintura de Fattorusso hacia Giordano haya sido provocado por Giuseppe Simonelli, con quien había colaborado en 1698. Sin embargo, en el movimiento fragmentado de las pinceladas robustas, se puede todavía reconocer algunas reminiscencias de Beinaschi.

Si no fuera por la verificación de la firma en relación con la otra firma presente en el S. Eugenio de la iglesia de Regina Coeli en Nápoles, no me atrevería a identificar al autor de la tela de Mugnano con Giuseppe Fattorusso, tanto por razones estilísticas como por la cronología tan tardía. Sin embargo, persiste la duda de que pudiera ser otro pintor, a lo mejor hijo de Giuseppe, del cual se han perdido las huellas.

La biografía de Giuseppe Fattorusso presenta muchas lagunas: De Dominici ${ }^{20}$ lo recuerda como alumno antes de Andrea Vaccaro y luego de Giovan Battista Beinaschi. Sus pinturas al óleo o al fresco eran visibles en Nápoles: en la iglesia del Carmine Maggiore («... realizó muchas obras al fresco, como se puede ver en las historias pintadas en la iglesia del Carmine maggiore sobre las de Luigi Siciliano, donde representó la vida de nuestro Señor»), en la iglesia de S. Pietro in Vinculis ( «... donde las Historias de S. Pedro, y todo lo que está al fresco, exceptuando la luneta sobre el altar, es de Fattoruso ), en la iglesia de S. Giuseppe dei Falegnami («... encontrándose todavía bajo la dirección del Vaccaro modificó las Historias de la Vida de S. José ... que eran obras de Simone Papa el joven) y en la iglesia de S. Diego all' Ospedaletto («... los cuadros al óleo, que adornan la Capilla de la B. Virgen en el coro, y la sacristía »).

Respecto a las pinturas de S. Giuseppe dei Falegnami o de los Carpinteros, visibles hasta la demolición de la iglesia en el año 1932, nos informa Galante ${ }^{21}$ que en la cúpula se encontraba pintado al fresco el Paraíso, "pintado por el Papa joven, modificado después por Fattorusso, y últimamente por Melchiorre de Gregorio».

Sin embargo, respecto a las pinturas de $\mathrm{S}$. Pietro in Vinculis, Antonio Delfino ha hallado un acta notarial en la cual Giuseppe Fattorusso

promete, y se obliga a pintar todos los cuadros, ángulos, triángulos, lunetas, et vacantes de la dicha Iglesia de San Pietro, a excepción de las cornisas, entonces ahí pintar tres grandes cuadros in primis la caída de Simón Mago, segundo la Anunciación, et argentum non est mihi, tercero la predica a la salida del Cenáculo, en las seis lunetas pintar seis pontífices, en el ángulo de la vidriera pintar aquello que parecerá al Muy Reverendo Señor D. Nicolò Maria Basile Rector de dicha Iglesia, en los doce triángulos pintar doces amorcillos, juegos, et en los lados de las vidrieras pintar los doce apóstoles. Ésta pintura dicho Gioseppe promete hacer, et acabar con cada perfección, et de aquella calidad, que Gioseppe ha pintado en la cúpula de dicha Iglesia, et aquella acabar el veinticinco de julio primo venturo del presente año $1676^{22}$.

Otras noticias sobre la actividad de Fattorusso, relativas a obras perdidas, provienen de fuentes documentales. Giovan Battista d'Addosio $^{23}$ encontró un pago de 1670 a Giuseppe Fattorusso y Domenico Viola para la «pintura de los Ss. Patronos [Pedro y Gennaro] ... en la fachada de la Puerta Mayor de dicho hospedaje [de S. Gennaro dei Poveri]» y para un cuadro de S. Pedro en la homónima capilla en el interior de la iglesia anexa. El mismo D'Addosio encontró dos pagos a Fattorusso, uno de 1660 y otro de 1671, para la restauración de distintas pinturas en la iglesia de la Annunziata. Otros documentos, destacados por Antonio Delfino, se centran en la ejecución de diez cuadros redondos para la congregación de los Siete Dolores en S. Spirito di Palacio, en el $1683^{24}$, y de algunas pinturas en la capilla de S. Cataldo en la ex catedral de Massalubrense, en el $1687^{25}$. En el mismo año, 
como atestigua un documento descubierto por Renato Ruotolo ${ }^{26}$, Fattorusso pintó un cuadro para la duquesa de Sicignano.

Encontramos finalmente un pago del año 1698 al escultor Giuseppe Troccola ${ }^{27}$, para un busto en plata de S. Antonio abad, para colocar en la capilla del tesoro de S. Gennaro ${ }^{28}$. Troccola había ejecutado cuatro modelos en creta, y una comisión de expertos, formada por los pintores Domenico Viola, Giuseppe Fattorusso y Giuseppe Simonelli, había escogido el mejor.

Hoy no quedan más que los frescos de la cúpula de S. Pietro in Vinculis, que representan el Paraíso ${ }^{29}$, y la tela con S. Eugenio I papa en S. Maria Regina Coeli para testimoniar la producción de Fattorusso en las iglesias de Nápoles. En los frescos de S. Pietro in Vinculis, que se pueden datar sobre base documental antes del final de 1680, aparece la influencia determinante de Beinaschi por el relieve plástico, la intensa luminosidad, la audaz visión de escorzo, y la composición dinámica y barroca. Como en el Paraíso del Beinaschi en los Ss. Apóstoles, también aquí asistimos a la progresiva anulación del peso corpóreo, que en la parte inferior es subrayado por un marcado claroscuro, mientras que en el vértice se disuelve en una intensa luminosidad. A propósito, es significativo que Galante adscriba la obra a Beinaschi, «no obstante otros [De Dominici, n.d.r.] la atribuyen a Giuseppe Fattorusso» ${ }^{30}$.

La sustancial influencia de Beinaschi se confirma en S. Eugenio / de Regina Coeli (Fig. 14), firmado «Fatteruso F. MDCLXXVI», obra de una serie de 14 lienzos que se encuentran alrededor de la nave centra ${ }^{31}$. La potente figura del santo pontífice llena toda la superficie del lienzo mixtilíneo. En su parte inferior se encuentra una placa pintada con el nombre del personaje representado, la firma y la fecha. El S. Eugenio / es acompañado por una S. Ripsima virgen y mártir, datada MDCLXXV, que Nicola Spinosa ${ }^{32}$ atribuía a Beinaschi, pero que, observando bien, se puede adscribir al mismo Fattorusso. En efecto, aunque el enfoque de la figura y la factura de los pliegues se inspiran en los modos de Beinas- chi influenciado por Lanfranchi, sin embargo la rígida postura de las manos y la excesiva regularidad del rostro, privado de pathos, denuncian la ejecución de un alumno, identificable como Fattorusso. Una confrontación con la Virgen Anunciada de Beinaschi que apareció en Sotheby's en Londres (lote n. 132 del 19/04/89) evidencia, por una parte, las afinidades tipológicas y compositivas, y por otra, la diferente calidad de la ejecución.

Podemos adscribir a Fattorusso también otro lienzo de la serie, S. Begga viuda, cuya datación no es legible ya que está escondida por el marco. La S. Begga presenta elementos muy semejantes a la S. Ripsima en el enfoque clasicista, en la soltura de los pliegues y en el rostro redondeado, demasiado regular y soso para poder sostener una atribución al maestro del Piamonte.

El análisis de las obras conocidas hace todavía más problemática la identificación del pintor de Mugnano con Giuseppe Fattorusso, pero lo que aquí interesa destacar es que la tela de Mugnano se puede considerar uno de los más importantes testimonios de la influencia de Giordano en la provincia de Nápoles.

\section{Post scriptum}

Durante los trámites para la publicación de este artículo he tenido la oportunidad de acceder y analizar dos telas históricamente atribuidas a Luca Giordano, provenientes de la capilla de Villa Figliola en S. Sebastiano al Vesuvio (Nápoles). Las dos telas representan el Éxtasis de $S$. Francisco de Asís y a S. Nicolás de Bari con el copero Basilio (Figs. 15 y 16); habían sido atribuidas por Prota-Giurleo ${ }^{33}$ en el año 1941, y superficialmente catalogadas por la Soprintendenza como obras de Luca Giordano. Con más prudencia, en la monografía dedicada al maestro por Scavizzi y Ferrari ${ }^{34}$ las mencionaban con reservas, al no haber podido estudiarlas en vivo. Hoy, tras haber tenido finalmente la oportunidad de inspeccionarlas, concluyo que las dos telas no pueden adscribirse a Luca Giordano, sino más bien a un desconocido seguidor de mediados del Setecientos. 
NOTAS

* Traducción: Emanuela Saladini.

${ }^{1}$ B. De Dominici, Vite de' pittori, scultori ed architetti napoletani, Napoli, 1742-45, t. III, pp. 441-453.

2 O. Ferrari - G. Scavizzi, Luca Giordano, Napoli, 1966, vol. II, pp. 103-104; vol. III, fig. 180.

${ }^{3}$ G. Parente, Origini e vicende ecclesiastiche della città di Aversa, Napoli, 1858, vol. II, p. 247.

${ }^{4} \mathrm{G}$. Labrot, Études napolitaines. Villages, palais, collections XVI-XVIII siècles, Seyssel, 1993, p. 240.

${ }^{5}$ O. Ferrari - G. Scavizzi, Luca Giordano, Napoli, 1966, vol. III, figg. 205-206.

${ }^{6}$ M. Pasculli Ferrara, "Giordano, Miglionico ed altri episodi giordaneschi", in Ricerche sul '600 napoletano, Napoli, 1991, p. 212 e fig. 107 p. 229.

${ }^{7}$ M. Izzo, Nicola Vaccaro (16401709), investigación de doctorado en "Metodologías cognoscitivas para la conservación y la valorización de los bienes culturales", XV ciclo, Seconda Università degli Studi di Napoli, Facultad de Letras y Filosofía, aa. 20002002, pp. 96-97.

${ }^{8}$ M. Izzo, Nicola Vaccaro (16401709). Un artista a Napoli tra Barocco e Arcadia, Todi, 2009, p. 249, sch. C7.

${ }^{9}$ S. Causa, "Quel che resta di un capolavoro perduto", Kronos, n. 9, 2005, pp. 51-113. El historiador parte del análisis de un fragmento de la Galería Regional de Sicilia para analizar las distintas versiones de la Bendición de Jacob que siguen a Giordano. Una reciente interpretación del tema ha aparecido en Nápoles en el año 2008 en una subasta de Blindarte (lote n. 47 del 20/05/2008).

${ }^{10}$ Óleo sobre tela, 73,5 x $99 \mathrm{~cm}$.

${ }^{11}$ B. De Dominici, Vite de' pittori, scultori ed architetti napoletani, Napoli, 1742-45, t. III, p. 445.

${ }^{12} \mathrm{Cfr}$. M. di Mauro en P. Leone de Castris (a cura di), Il Museo Diocesano di Napoli. Percorsi di arte e di fede, Nápoles 2008, p. 152, sch. 54.
${ }^{13}$ Cfr. O. Ferrari - G. Scavizzi, Luca Giordano, Napoli, 1966, II, p. 110, III, fig. 188.

${ }^{14}$ Cfr. O. Ferrari - G. Scavizzi, Luca Giordano, Napoli, 1966, II, p. 168, III, fig. 331.

15 M. di Mauro, In viaggio. La Campania. Ricerche e attribuzioni alla scoperta delle opere e degli artisti, Napoli, 2009, p. 66.

${ }^{16} \mathrm{Cfr}$. V. Lotoro, "Una replica del De Matteis dell'Adamo ed Eva che piangono Abele morto", in F. Abbate (a cura di), Percorsi di conoscenza e tutela. Studi in onore di Michele D'Elia, Napoli, 2008, nota 26, p. 288.

${ }^{17} \mathrm{Cfr}$. U. Di Furia, "I Sarnelli: una famiglia di pittori e di bancari", Quaderni dell'Archivio Storico, Napoli, 2005-2006, pp. 268-269.

${ }^{18} \mathrm{~V}$. Pacelli in /l Museo del Barocco Romano. Le collezioni Ferrari, Laschena ed altre donazioni a Palazzo Chigi in Ariccia, Ariccia, 2008, pp. 50-51.

${ }^{19} \mathrm{Cfr}$. N. Spinosa, Pittura napoletana del Settecento. Dal Barocco al Rococò, Napoli, 1986, sch. 200, p. 150; fig. 237 p. 306.

${ }^{20}$ B. De Dominici, Vite de' pittori, scultori ed architetti napoletani, Napoli, 1742-45, t. III, pp. 156, 283.

${ }^{21}$ G.A. Galante, Guida sacra della città di Napoli, Napoli, Stamperia del Fibreno, 1872, p. 330.

${ }^{22}$ A. Delfino, "Documenti inediti tratti dall'Archivio di Stato di Napoli (A.S.N.) e dall'Archivio Storico del Banco di Napoli (A.S.B.N.)", in Ricerche sul '600 napoletano, Napoli, 1993, doc. 21, p. 31.

${ }^{23}$ G.B. D'Addosio, Documenti inediti di artisti napoletani dei secoli XVI e XVII dalle polizze dei Banchi, Napoli, 1920, p. 53.

${ }^{24}$ A. Delfino, "Documenti inediti su alcuni pittori napoletani del Seicento", in Ricerche sul '600 napoletano, Napoli, 1984, p. 158.

${ }^{25}$ A. Delfino, "Documenti inediti per alcuni pittori napoletani del '600", in Ricerche sul '600 napoletano, 1985, p. 100. Hoy la capilla aparece decora- da por estucos del Novecientos, bajo los cuales se podrían encontrar las pinturas de Fattorusso.

${ }^{26}$ R. Ruotolo, "Documenti sulla chiesa di S. Teresa agli Studi e su qualche pittore napoletano del Seicento", in Ricerche sul '600 napoletano, 1983, p. 63.

${ }^{27}$ Arch. Storico Ist. Banco de Nápoles Fundación (desde ahora ASBN), Banco dei Poveri, periódico c.p. de caja, 1698 - II sem., matr. 741, partida de 20 ducados extinguida el 14 de noviembre: «A Matteo Carbone ducados 20 y por ésto a Giuseppe Troccola escultor de mármoles, dijo al acabar de ducados 48 , esperando del otro de éste ha recibido en contantes, dijo pagarlos por sus fatigas hechas en la formación de dos modelos pequeños de creta y dos grandes de la misma materia del glorioso S. Antonio de Viena, que a sus súplicas ha sido declarado por uno de los padrones e protectores de esta muy fiel Ciudad, de lo último de los cuales se tendrá que formar la estatua de medio tronco de plata de dicho Santo para conservarse en el Tesoro de esta Ciudad, y proprio el modelo reconocido, et aprobado por el Cav. Domenico Viola, y Giuseppe Fattoruso, y Giuseppe Simonelli pintores, et expertos, por ésto Matteo diputados para la visura de dicho modelos, quedando dicho Giuseppe enteramente satisfecho...» (una parcial transcripción del documento se encuentra en V. Rizzo, "Uno sconosciuto paliotto di Lorenzo Vaccaro e altri fatti coevi napoletani", en Storia dell'Arte, 49, 1983, pp. 222-223).

${ }^{28}$ El busto de S. Antonio Abate que actualmente se venera en la Capilla del Tesoro es obra del siglo XIX.

29 Doy las gracias a Patrizia Di Maggio por mostrarme una fotografía de la cúpula, dado que la iglesia está actualmente fuera de uso y en estado de devastación. No sé que quedará de las otras pinturas aquí ejecutadas por Fattorusso y listadas en el citado acta notarial (A. Delfino, "Documenti inediti tratti dall'Archivio di Stato di Napoli (A.S.N.) e dall'Archivio Storico del 
Banco di Napoli (A.S.B.N.)", in Ricer-

che sul '600 napoletano, 1993, doc. 21 p. 31).

${ }^{30}$ G.A. Galante, Guida sacra della città di Napoli, Napoli, Stamperia del Fibreno, 1872, p. 313.

${ }^{31}$ Cfr. B. Daprà (a cura di), Micco
Spadaro. Napoli ai tempi di MasanieIlo, catalogo de la exposición (Napoli, Certosa di S. Martino, 20 apr.-30 giu. 2002), Napoli, 2002, p. 170. Cada una de las 14 telas mide $200 \times 110 \mathrm{~cm}$.

${ }^{32}$ Cfr. G.A. Galante, Guida sacra della città di Napoli, 1872, ed. critica a cura di N. Spinosa, Napoli, 1985, p. 64.

${ }^{3}$ U. Prota-Giurleo, "Notizie inedite su Luca Giordano", Corriere di Napoli, 3 maggio 1941; reeditado en Napoli. Rivista municipale, 1957.

${ }^{34}$ O. Ferrari - G. Scavizzi, Luca Giordano, Napoli, 1966, t. II, p. 382.

\title{
TEXTO ORIXINAL
}

\author{
Inediti e considerazioni su Paolo de Matteis, Giuseppe Simonelli, \\ Lorenzo Ruggi ed altri Giordaneschi
}

Marco di Mauro

Università di Napoli

Sono innumerevoli i discepoli, seguaci e imitatori di Luca Giordano: il De Dominici' menziona decine di allievi, alcuni dei quali, come Franceschitto o Monsù Anselmo, sono ancora da identificare. Altri pittori giordaneschi sono noti unicamente dalle firme apparse su tele ed affreschi, in occasione di restauri o puliture che hanno rimosso la polvere che le occultava. Fra questi pittori ricordiamo Lorenzo Ruggi, che firma e data 1671 una tela con I'Immacolata nella chiesa di S. Francesco ad Aversa (fig. 1). Sulla data, però, nutro qualche perplessità, poiché il dipinto deriva da modelli giordaneschi come l'Immacolata del Museo de Arte di Ponce, o quella di Palazzo Pitti a Firenze (fig. 2), che si datano intorno al 1680 per le sensibili aperture classiciste².

L'opera di Lorenzo Ruggi manifesta la sua discendenza da modelli del Giordano nel tenero incarnato dei putti, nelle brillanti vesti di Maria, nella luce dorata che irrompe fra le nuvole, nella vitalità della composizione che viene accentuata dal vivace assembramento dei putti. È pertanto verosimile che Lorenzo Ruggi fosse uno «scolaro del Giordano», come afferma il Parente ${ }^{3}$, sebbene non sia annoverato dal De Dominici tra i suoi discepoli. Altre notizie ci sono fornite dal Labrot ${ }^{4}$, che cita, sulla base dei documenti d'archivio, opere di Lorenzo Ruggi negli inventari di due collezioni napoletane: quella del dottore Antonio Lauro, suo fratellastro, e quella del principe di Montefalcone Andrea Coppola. II Ruggi, morto nel 1687, avrebbe eseguito in prevalenza soggetti sacri, ma anche paesaggi di cui però non rimane traccia. Labrot ipotizza che sia morto in piena attività, poiché alla sua morte risultano esservi molti quadri invenduti nella sua bottega.

A differenza del Ruggi, Giuseppe Simonelli può vantare una cospicua bibliografia e centinaia di opere firmate o attribuite. Tra gli ultimi ritrovamenti si annovera l'Apoteosi di S. Giovanni Evangelista nella chiesa di S. Giovanni a Teverola (fig. 3). Fino al 1989, quando la tela fu rimossa dal soffitto della navata per sottoporla a restauro, si riteneva opera di un ignoto napoletano, ma la scoperta della firma, in basso a destra, non lascia dubbi 